\title{
REVIEW
}

\section{Syncope in childhood}

\section{K A McLeod}

Syncope in childhood is very common. The vast majority of episodes are benign, and are due to neurocardiogenic syncope. Only a minority are due to something potentially more serious or life threatening. The diagnosis and differentiation of benign from more serious causes of syncope is made primarily by the history. Investigations are often unfruitful. The mainstay of management in neurocardiogenic syncope is reassurance. An increase in dietary fluid and salt can be helpful. Drug treatment is reserved for those with more frequent and severe attacks. Cardiac pacemakers should be reserved for those with very severe symptoms who are refractory to drug therapy.

U to one in five children experience an episode of syncope before the age of 15 years. Whereas the vast majority of episodes of syncope are benign, a minority are caused by something potentially more serious or even life threatening. Even benign syncope has a death like quality. ${ }^{1}$ It thus tends to generate extreme anxiety and is often extensively, inappropriately, and unfruitfully investigated.

\section{CLASSIFICATION}

The causes of childhood syncope can broadly be divided into three groups, the third group consisting of syncope look-alikes or pseudosyncopes (table 1). ${ }^{2}$ Neurally mediated syncopes are benign causes of syncope, which occur when there is a disturbance in the autonomic nervous system's control of heart rate and blood pressure. Cardiovascular causes of syncope are rare in childhood, but are potential causes of sudden death. Non-cardiovascular pseudo-syncopes can

Table 1 Classification of syncope in childhood

Neurally mediated syncopes

Reflex syncopes

Postural orthostatic tachycardia syndrome (POTS)

Pure autonomic failure

Multiple system atrophy

Correspondence to:

Dr K A McLeod, Consultant

in Paediatric Cardiology,

Department of Cardiology,

Royal Hospital for Sick

Children, Yorkhill NHS

Trust, Glasgow G3 8SJ

UK; karen.mcleod@

yorkhill.scot.nhs.uk

Accepted 23 July 2002

Arrhythmic

Structural

Vascular

Non-cardiovascular pseudo-syncopes

Epileptic

Psychogenic broadly be divided into the epilepsies and the psychogenic causes in which the syncope is factitious. ${ }^{3}$ The aim of this review will be to concentrate on the commonest form of syncope in childhood, the neurally mediated syncopes.

\section{NEURALLY MEDIATED SYNCOPES}

Neurally mediated syncopes are a heterogeneous group of autonomic disorders, which result in orthostatic intolerance. ${ }^{4}$ They can be divided into four main groups (table 1). Postural orthostatic tachycardia syndrome, pure autonomic failure, and multiple system atrophy are all types of chronic autonomic failure, whereas reflex syncopes are transient disturbances in autonomic control of heart rate and blood pressure. ${ }^{4-6}$

Neurocardiogenic syncope can be considered as a form of reflex syncope and is by far the commonest type of syncope in childhood. It can occur at any age, but the peak age groups are toddlers and older children between the ages of 9 and 14 years. A typical history in an older child is of syncope that occurs when the child is upright, either sitting or standing. Characteristically there is a prodrome such as dizziness, nausea, and pallor, before loss of tone and consciousness. Depending on the duration and severity of cerebral hypoxia secondary to hypotension and/or profound bradycardia, the child may have an anoxic seizure and may be incontinent. An anoxic seizure is different from an epileptic seizure in that the electroencephalogram (EEG) is flat, and rather than tonic-clonic movements, there tends to be stiffening, opisthotonus, and fine twitching. ${ }^{7}$ On recovery the child may feel tired and "washed out" for some time. In toddlers, reflex syncope tends to manifest as so called "reflex anoxic seizures" or "pallid breath holding spells" with reflex asystole followed by an anoxic seizure in response to a noxious stimulus (fig 1A). ${ }^{8}$ Of note is that the distinction between "pallid breath holding" and "blue breath holding" is more blurred than often realised. Both are types of reflex syncope, but the mechanism behind the cyanosis is poorly understood. ${ }^{89}$

\section{DIAGNOSIS OF SYNCOPE}

Investigations for syncope in childhood will almost always be normal, and the key to the diagnosis is a detailed and careful history. When a careful history is taken, it will usually be clear to which of the three categories the syncope belongs. In the vast majority of cases, the syncope will be neurally mediated. There are, however, a

Abbreviations: $E C G$, electrocardiogram; EEG, electroencephalogram 
A

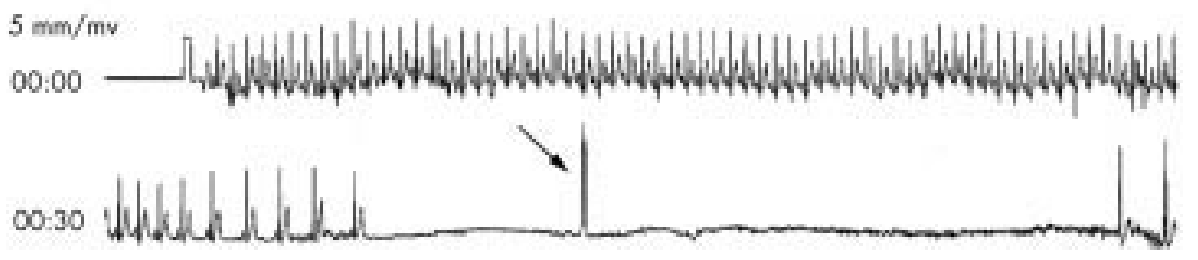

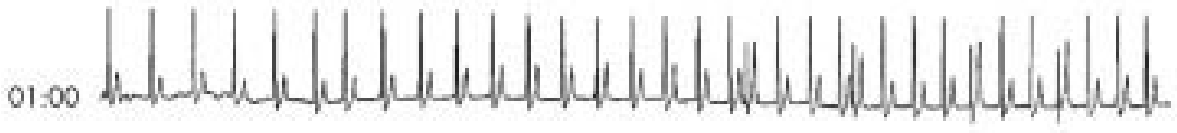

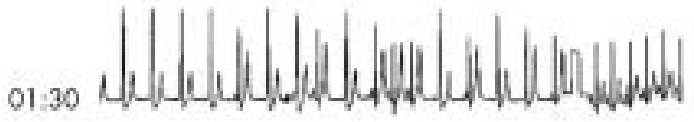

B

$5 \mathrm{~mm} / \mathrm{mv}$

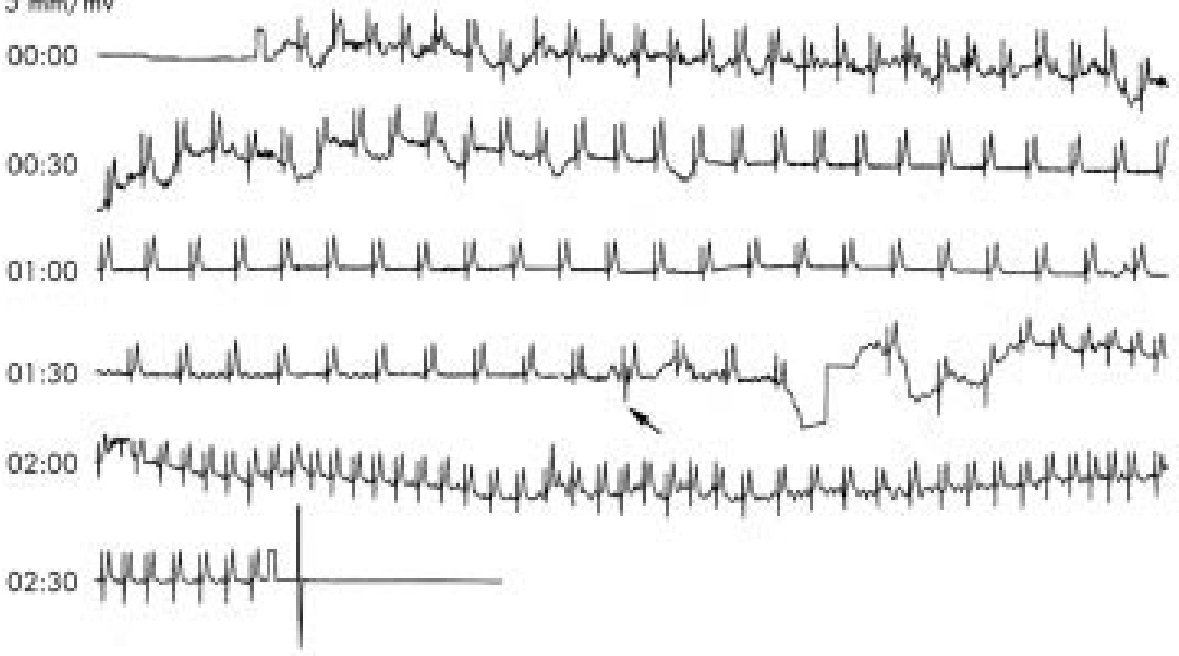

Figure 1 (A) ECG recording from a 2 year old child during a reflex anoxic seizure. Each strip corresponds to 30 seconds of recording. The rhythm is initially sinus tachycardia corresponding to the child running. Following a bump to the elbow, the heart slows considerably to a period of asystole, lasting approximately 16 seconds. The arrow shows the point at whicih the monitor was activated-within seconds of the onset of ECG changes. (B) ECG recording from an 8 month old child in whom syncope was induced by smothering. Each strip corresponds to 30 seconds of recording. The parent was asked to activate the monitor at the onset of symptoms. The arrow shows the point at which the monitor was activated, but it is evident that ECG changes of significant bradycardia are present well before the monitor is activated.

number of "warning bells" from the history that should indicate a potentially more serious or life threatening cause (table 2).

\section{INVESTIGATIONS FOR RECURRENT SYNCOPE}

Probably the most important investigation for recurrent syncope is a 12 lead electrocardiogram (ECG), primarily to exclude a long QT interval. Pre-excitation, heart block, or ventricular hypertrophy can also be diagnosed from an ECG. Although the ECG will almost always be normal in children with recurrent syncope, a normal ECG is an important finding. In older children, if symptoms are related to exercise,

Table 2 Syncope "warning bells"

Syncope in response to loud noise, fright, or extreme emotional stress Syncope during exercise

Syncope while supine

Family history of sudden death in young person $<30$ years old Syncope with an "odd" history an exercise test should be performed in the hope of inducing symptoms. In reality, symptoms rarely occur during the test. Holter monitoring is usually unhelpful, as symptoms almost never occur in the 24-48 hour period while the monitor is worn. Cardiac event monitoring has a theoretical advantage over Holter monitoring in that the monitors can be worn for a longer period of time, such as weeks or months. The aim is to try to record the ECG during symptoms, but inevitably the monitor never seems to be worn at the time an event occurs. Unless the child has other cardiac signs or symptoms, or any of the warning bells from the history, an echo will almost certainly be normal. Although an EEG is often performed on children with syncope to "exclude epilepsy", this is rarely helpful. Even in children with epilepsy the EEG will usually be normal between attacks, and a percentage of children without epilepsy will have a frankly "epileptic" EEG. Intracerebral causes of syncope are very rare in childhood and would usually be associated with other neurological signs or symptoms; thus magnetic resonance imaging or computed tomography scan is usually an expensive waste of time.

A suggested approach to the investigation of the child with recurrent syncope is as follows. A 12 lead ECG should be 
performed primarily to exclude a long QT. If there are any of the "warning bells" from the history or if there are any other cardiac or neurological signs or symptoms, appropriate cardiac or neurological investigations should be undertaken. If there is a good history for neurally mediated syncope and the ECG is normal, usually no further investigation is required. Further investigation may be considered for those who have very severe or frequent attacks, who are in need of reassurance, or where the history is not entirely clear. Where there is a good history for neurally mediated syncope, tilt testing is probably the most productive investigation for older children, and cardiac event monitoring for the toddler.

\section{TILT TESTING}

Tilt testing can be successfully performed in children from the age of 6 years. There is no standardised protocol for tilt testing and protocols vary in terms of duration of tilt, degree of tilt, and whether or not drugs such as isoprenaline are given. ${ }^{10}$ Our own protocol is very simple and tolerated by most children. The child rests supine for 15 minutes and is then tilted to 60 degrees head-up for a maximum of 45 minutes. During this time the blood pressure is continuously but non-invasively monitored using the Finapres system, and a three lead ECG continuously recorded. The main "side effect" of the test is boredom, and younger children especially have a tendency to become restless. Using this protocol, approximately $50 \%$ of children with a good history for neurally mediated syncope will have a positive tilt test. The use of drugs increases the sensitivity of the test, but reduces its specificity and makes the test more unpleasant for the child. Whatever tilt test protocol is chosen, the three important requirements appear to be a period of supine rest before tilting, to tilt to between 60 and 80 degrees to reduce false positive and negative responses, and to tilt for at least 40 minutes of drug free period. ${ }^{1}$

In those who have a positive tilt test, the most common response is a combination of hypotension and bradycardia prior to syncope. Hypotension with no significant change in heart rate is the next most common positive response. The least common positive response is asystole prior to syncope, often termed a "cardioinhibitory" response.

Another type of reflex syncope, which has only been recognised relatively recently, is cerebral vasoconstrictive syncope. ${ }^{12}$ In this condition syncope is caused by cerebral vasoconstriction without hypotension and bradycardia. Recording an EEG or measuring cerebral blood flow during a tilt test in individuals who have symptoms despite normal blood pressure and heart rate should help make the diagnosis.

In situations where it is claimed that syncope is occurring several times a day every day, admission to hospital for observation with continuous ambulatory ECG and EEG monitoring is usually the best approach. Almost always the ECG, EEG, and measured blood pressure will be found to be normal during the "syncopes" and a diagnosis of psychogenic pseudosyncope can be confirmed.

For toddlers with reflex asystolic syncopes, cardiac event monitoring can help confirm diagnosis, although an ECG recording during symptoms is only likely to be obtained in those few who have very frequent attacks (fig lA).

In infants who have recurrent and severe episodes of syncope that only have their onset in the presence of a particular parent or guardian, factitious or induced illness should be considered. This is particularly likely if some of the syncopes are shown to other adults-relatives or hospital staff-before the infant has recovered..$^{13}{ }^{14}$ The child should be admitted to hospital for continuous ambulatory EEG monitoring together with cardiac event monitoring and overt video surveillance. ${ }^{15}$ The parent or guardian should be asked to activate the cardiac event monitor at the onset of any syncopal event. Typically in the situation of induced syncope e.g. by smothering, there tends to be a significant delay between the

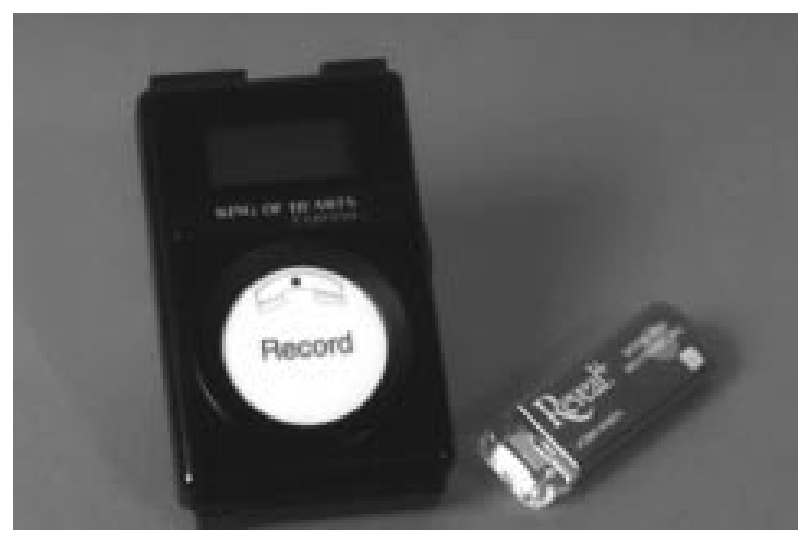

Figure 2 Cardiac event monitoring allows the recording of an ECG during symptoms. Non-invasive monitors such as the King of Hearts can be worn for weeks or even months at a time. The implantable Reveal monitor (Medtronic Inc.) is useful where monitoring is required for a prolonged period of time or where compliance is poor. It is easily implanted in a small subcutaneous pocket and allows monitoring for up to 14 months.

onset of EEG and ECG changes and activation of the monitor (fig 1B). ${ }^{16}$ This is different from most spontaneous syncope episodes, where activation of the monitor tends to occur within seconds of the onset of ECG and EEG changes (fig 1A).

Occasionally the distinction between neurally mediated syncope and potentially serious arrhythmia remains unclear, for example, in a child with a borderline QT interval or slightly worrying history. In this situation, an implantable Reveal monitor (Medtronic Inc., USA) can be useful (fig 2).

\section{MANAGEMENT OF NEURALLY MEDIATED SYNCOPE}

The mainstay of therapy is reassurance, specifically that the episodes are not caused by epilepsy or a cardiac problem. ${ }^{17}$ Advice should be given to drink plenty and to increase dietary salt. When prodromal symptoms are experienced, manoeuvres such as crossing the legs and folding the arms, especially when standing, help to maintain blood pressure. Often with the above simple measures of reassurance, fluid, posture, and salt, symptoms will improve significantly. In toddlers with reflex asystolic syncope, parents should be advised to place the child in the recovery position and avoid the natural tendency to pick up the child.

The likelihood of further syncopal attacks depends on the number of episodes of syncope prior to presentation. ${ }^{18}$ For those who present with frequent syncope or continue to have syncope despite the above simple measures, drug therapy should be considered. There are many pharmacological agents available, but no drug has been adequately evaluated by randomised clinical trials. Probably all have a degree of placebo effect. Fludrocortisone and $\beta$ blockers are the most favoured first line drugs, with relatively few side effects. ${ }^{19}$ A reasonable first approach is fludrocortisone at a dosage of $100 \mu \mathrm{g} / \mathrm{day}$. This is effective in most children in reducing frequency and severity of syncope. Occasionally the drug is not tolerated as a result of fluid retention and weight gain. If symptoms continue despite fludrocortisone, addition of a $\beta$ blocker can be helpful. Serotonin uptake inhibitors such as fluoxetine hydrochloride, and vasoconstrictors such as midodrine, an $\alpha$ agonist, are currently under clinical review, but their safety has not been established in children. ${ }^{20}{ }^{21}$ It would seem sensible to reserve them for those who continue to have symptoms despite first line therapies. An alternative or adjunct to drugs is biofeedback techniques, including tilt training and active tension. ${ }^{22}$ For toddlers with neurocardiogenic syncope, iron therapy might be helpful. ${ }^{23}$ Iron deficiency may be more important in cyanotic rather than pallid breath holding spells, 
but there is often overlap between the two. Nevertheless it is reasonable to look for and treat any iron deficiency in toddlers with neurocardiogenic syncopes. Atropine can be effective in reducing severity and frequency of syncope, but side effects, including loss of concentration, dry mouth, gastrointestinal upsets, and behavioural problems are common. ${ }^{24}$

\section{CARDIAC PACING}

The use of cardiac pacing for neurocardiogenic syncope remains controversial. ${ }^{25}$ The idea behind pacing is that it should prevent any signficant bradycardia or asystole that might contribute to the hypotension that ultimately results in syncope. It would not be expected to prevent hypotension that is caused by vasodilatation. There is little evidence, however, that profound bradycardia occurs during most spontaneous attacks, even if it occurs during tilt testing.

A number of studies have shown a benefit from pacing for neurocardiogenic syncope, but only one of the studies successfully addressed the issue of placebo. ${ }^{26-28}$ As cardiac pacing is a significant commitment in a young person, it should be reserved for those who have severe, frequent attacks and in whom drug therapy has failed or is declined. While the question remains as to whether children with neurocardiogenic syncope but without demonstrable asystole will benefit from pacing, it would seem sensible to reserve pacing for those who have a recorded aystole or profound bradycardia during a typical attack. One approach is to implant a Reveal monitor in any child with frequent neurally mediated syncope in whom a pacemaker is being considered. The Reveal allows a more accurate assessment both of the frequency of syncope and whether asystole or profound bradycardia occurs during spontaneous syncope. It has been our experience that implantation of the Reveal can in itself have an astonishingly curative effect, perhaps by working as a placebo or as a form of biofeedback, and may even circumvent the need for a pacemaker.

\section{REFERENCES}

1 Engel GL. Psychological stress, vasodepressor syncope and sudden death. Ann Intern Med 1978;89:403-12.

2 Ross B, Grubb B. Syncope in the child and adolescent. In: Grubb B Oshlansky B, eds. Syncope: mechanisms and management. New York: Futura, 1998:305-16.

3 Mathias CJ, Deguchi K, Beasedale-Barr K, et al. Familial vasovagal syncope and pseudosyncope: observations in a case with both natura and adopted siblings. Clin Auton Res 2000;10:43-5.

4 Grubb BP, Karas B. Clinical disorders of the autonomic nervous system associated with orthostatic intolerance: an overview of classification, clinical evaluation, and management. PACE 1999;22:789-810.

5 Grubb BP. Pathophysiology and differential diagnosis of neurocardiogenic syncope. Am J Cardiol 1999;84:3Q-9Q.
6 Karas B, Grubb BP, Boehm K, et al. The postural orthostatic tachycardia syndrome: a potentially treatable cause of chronic fatigue, exercise intolerance, and cognitive impairment in adolescents. Pacing Clin Electrophysiol 2000;23:344-51.

7 Stephenson JBP. Nonepileptic seizures, anoxic-epileptic seizures and epilpetic-anoxic seizures. In: Wallace S, ed. Epilepsy in children. London: Chapman and Hall, 1996:5-26.

8 Stephenson J, McLeod K. Reflex anoxic seizures. In: David TJ, ed. Recent advances in paediatrics. Edinburgh: Churchill Livingstone, 2000:1-19.

9 Breningstall GN. Breath-holding spells. Pediatr Neurol 1996;14:91-7.

10 Sutton R, Bloomfield D. Indications, methodology, and classification of tilt table testing. Am J Cardiol 1999:84:10Q-19Q.

11 Kenny RA, O'Shea D, Parry SW. The Newcastle protocols for head-up tilt table testing in the diagnosis of vasovagal syncope and related disorders. Heart 2000;83:564-9.

12 Grubb B. Cerebral syncope: new insights into an emerging entity. J Pediatr 2000;136:431-2.

13 Rosen CL, Frost JD Jr, Glaze DG. Child abuse and recurrent infant apnea. J Pediatr 1986;109:1065-7.

14 Stephenson JBP. Fits and faints. London: MacKeith Press, 1990.

15 Stephenson JBP. Video surveillance in diagnosis of intentional suffocation. Lancet 1994;344:414-15.

16 Hoorntie TM, Langerac W, Sreeram N. Munchausen's syndrome by proxy identified with an implantable electrocardiographic recorder. $N$ Engl J Med 1999;341:1478-9.

17 Bloomfield DM, Sheldon R, Grubb BP, et al. Putting it together: a new treatment algorithm for vasovagal syncope and related disorders. Am J Cardiol 1999;84:33Q-39Q.

18 Grimm W, Degenhardt M, Hoffman J, et al. Syncope recurrence can be better predicted by history than by head-up tilt testing in untreated patients with suspected neurocardiogenic syncope. Eur Heart J 1997:18:1465-9.

19 Benditt D, Fahy G, Lurie K, et al. Pharmacotherapy of neurocardiogenic syncope. Circulation 1999;100:1242-8.

20 Di Girolamo E, Di lorio C, Sabatini P, et al. Effects of paroxetine hydrochloride, a selective serotonin reuptake inhibitor, on refractory vasovagal syncope: a randomized double-blind, placebo-controlled study. J Am Coll Cardiol 1999;33:1227-30.

21 Ward C, Gray J, Gilroy J, et al. Midodrine: a role in the management of neurocardiogenic syncope. Heart 1998;79:45-9.

22 Reybrouck T, Heidbuchel H, Van de Werf F, et al. Tilt training: a treatment for malignant and recurrent neurocardiogenic syncope. Pacing Clin Electrophysiol 2000;23:493-8.

23 Mocan H, Yildiran A, Orhan F, et al. Breath holding spells in 91 children and response to treatment with iron. Arch Dis Child 1999;81:261-2.

24 McWilliam RC, Stephenson JBP. Atropine treatment of reflex anoxic seizures. Arch Dis Child 1984:59:473-5.

25 Sheldon R. Role of pacing in the treatment of vasovagal syncope. Am J Cardiol 1999;84:26Q-32Q.

26 Connolly SJ, Sheldon RS, Roberts RS, et al. The North American Vasovagal Pacemaker Study: a randomized trial of permanent cardiac pacing for the prevention of vasovagal syncope. J Am Coll Cardiol 1999;33:16-20.

27 Villain E, Lucet V, Do Ngoc D, et al. Stimulation cardique dans les spasmes du sanglot de l'enfant. Archives des malades du Coeur et Vaisseaux 2000;547-52.

28 McLeod K, Wilson N, Hewitt J, et al. Double-blind trial of cardiac pacing for severe childhood neurally mediate syncope with reflex anoxic seizures. Heart 1999;82:721-6. 